\title{
Oxidation of Levitated Exo-Tetrahydrodicyclo- pentadiene Droplets Doped with Aluminum Nanoparticles
}

Michael Lucas $^{1 *}$, Stephen J. Brotton ${ }^{1 *}$, Ahreum Min ${ }^{1}$, Michelle L. Pantoya ${ }^{2}$, Ralf I. Kaiser ${ }^{1}$

${ }^{1}$ Department of Chemistry, University of Hawai'i at Manoa, Honolulu, HI 96822

${ }^{2}$ Mechanical Engineering Department, Texas Tech University, Lubbock, TX 79409

*Contributed equally to this work. 


\section{REACTION YIELDS}

$\mathrm{H}_{2} \mathrm{O}$ and $\mathrm{CO}_{2}$ are formed by oxidizing JP-10 through the reaction:

$$
\mathrm{C}_{10} \mathrm{H}_{16}+14 \mathrm{O}_{2} \rightarrow 10 \mathrm{CO}_{2}+8 \mathrm{H}_{2} \mathrm{O}
$$

In this section, we will determine the quantities of reactants and products for reaction (1) and the moles of unoxidized aluminum. Our process chamber has a volume of $15.44 \mathrm{~L}$ and was filled with $40 \pm 1 \%$ of $\mathrm{O}_{2}$. Therefore, using the ideal gas law, there were $0.2871 \pm 0.0053$ moles of $\mathrm{O}_{2}$ before reaction. The Al/JP-10 droplet studied here had a volume of $(4.6 \pm 1.4) \times 10^{-3} \mathrm{~cm}^{3}$ and contained 99.5 wt. \% of JP-10; therefore, using the density of $0.9314 \mathrm{~g} \mathrm{~cm}^{-3}$ for JP-10, ${ }^{1}$ it is determined that the unreacted droplet contained $(3.14 \pm 0.92) \times 10^{-5}$ mol of JP-10. Since the molar ratio of $\mathrm{O}_{2}: \mathrm{JP}-$ 10 of 9140:1 is much larger than the stoichiometric ratio of the reactants of 14:1 (see reaction (1)), $\mathrm{O}_{2}$ is the excess reactant. The FTIR spectrum in Figure 5 did not show evidence for $\mathrm{CO}$, which confirms that $\mathrm{O}_{2}$ was the excess reactant.

The oxygen can also react with the aluminum; the reaction for the complete oxidation of aluminum is:

$$
2 \mathrm{Al}+3 / 2 \mathrm{O}_{2} \rightarrow \mathrm{Al}_{2} \mathrm{O}_{3}
$$

The Al/JP-10 droplet contains 0.5 wt. \% of Al NPs with an average diameter of $80 \mathrm{~nm}$. The Al NPs have a $4 \mathrm{~nm}$ thick $\mathrm{Al}_{2} \mathrm{O}_{3}$ outer shell that needs to be accounted for when determining the initial quantity of unoxidized $\mathrm{Al}$. The mass ratio of $\mathrm{Al}$ to $\mathrm{Al}_{2} \mathrm{O}_{3}$ in the nanoparticle is therefore 1.839, where we have used $2.90 \mathrm{~g} \mathrm{~cm}^{-3}$ and $3.95 \mathrm{~g} \mathrm{~cm}^{-3}$ for densities of $\mathrm{Al}$ and $\mathrm{Al}_{2} \mathrm{O}_{3}$, respectively. The initial amount of unoxidized aluminum is consequently $(5.16 \pm 0.72) \times 10^{-7} \mathrm{~mol}$.

We can calculate the moles of $\mathrm{CO}_{2}$ and $\mathrm{H}_{2} \mathrm{O}$ produced by comparing the absorbances for $\mathrm{CO}_{2}$ and $\mathrm{H}_{2} \mathrm{O}$ observed in the FTIR spectrum (Figure 5) with the known cross sections. The required absorption cross sections for $\mathrm{CO}_{2}$ and $\mathrm{H}_{2} \mathrm{O}$ were calculating using the SPECTRA Information System $^{2}$ and the absorption lines from the HITRAN molecular spectroscopic database. ${ }^{3}$ The absorption cross sections were calculated for band $\mathrm{c}$ of $\mathrm{CO}_{2}$ centered at $2349 \mathrm{~cm}^{-1}$ and for band d of $\mathrm{H}_{2} \mathrm{O}$ at $1635 \mathrm{~cm}^{-1}$ (Figure 5, Table S3). The FTIR spectra of these bands and the reference absorption cross sections are shown in Figure S7. To calculate the moles of $\mathrm{CO}_{2}$ and $\mathrm{H}_{2} \mathrm{O}$ produced, the Beer-Lambert law was used:

$$
A=\frac{\mathrm{n} \sigma \mathrm{L}}{\log _{e}(10)}
$$


where $A$ is the absorbance, $\sigma$ is the absorption cross section, $L$ is the path length of $37.63 \mathrm{~cm}$, and $n$ is the number density of the absorber. At the maximum of band c for $\mathrm{CO}_{2}$ corresponding to 2362 $\mathrm{cm}^{-1}$, the measured absorbance is $0.283 \pm 0.001$ and the reference absorption cross section is $(2.608$

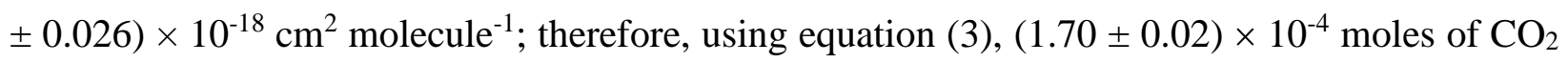
were produced. The quantity of $\mathrm{H}_{2} \mathrm{O}$ produced is calculated using the peak at $1653 \mathrm{~cm}^{-1}$, which reaches a maximum absorbance of $0.00887 \pm 0.00033$. The reference absorption cross section at

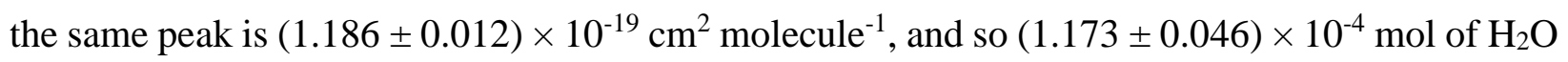
were produced. The molar ratio of $\mathrm{CO}_{2}$ to $\mathrm{H}_{2} \mathrm{O}$ is expected to be $1.25: 1$, whereas the measured molar ratio is $1.45: 1$ indicating that slightly less water was detected than expected. This could be caused by water condensing on the surfaces within the stainless-steel chamber. Therefore, we only use the quantity of $\mathrm{CO}_{2}$ formed to calculate the moles of $\mathrm{O}_{2}$ and $\mathrm{JP}-10$ consumed. The formation of $\mathrm{CO}_{2}$ would consume $(2.38 \pm 0.03) \times 10^{-4} \mathrm{~mol}$ of $\mathrm{O}_{2}$ and $(1.70 \pm 0.02) \times 10^{-5} \mathrm{~mol}$ of JP-10. The calculated amount of JP-10 consumed is a factor of 0.54 lower than the initial amount of JP-10 of $(3.14 \pm 0.92) \times 10^{-5} \mathrm{~mol}$, which is a consequence of the incomplete combustion of JP-10. 

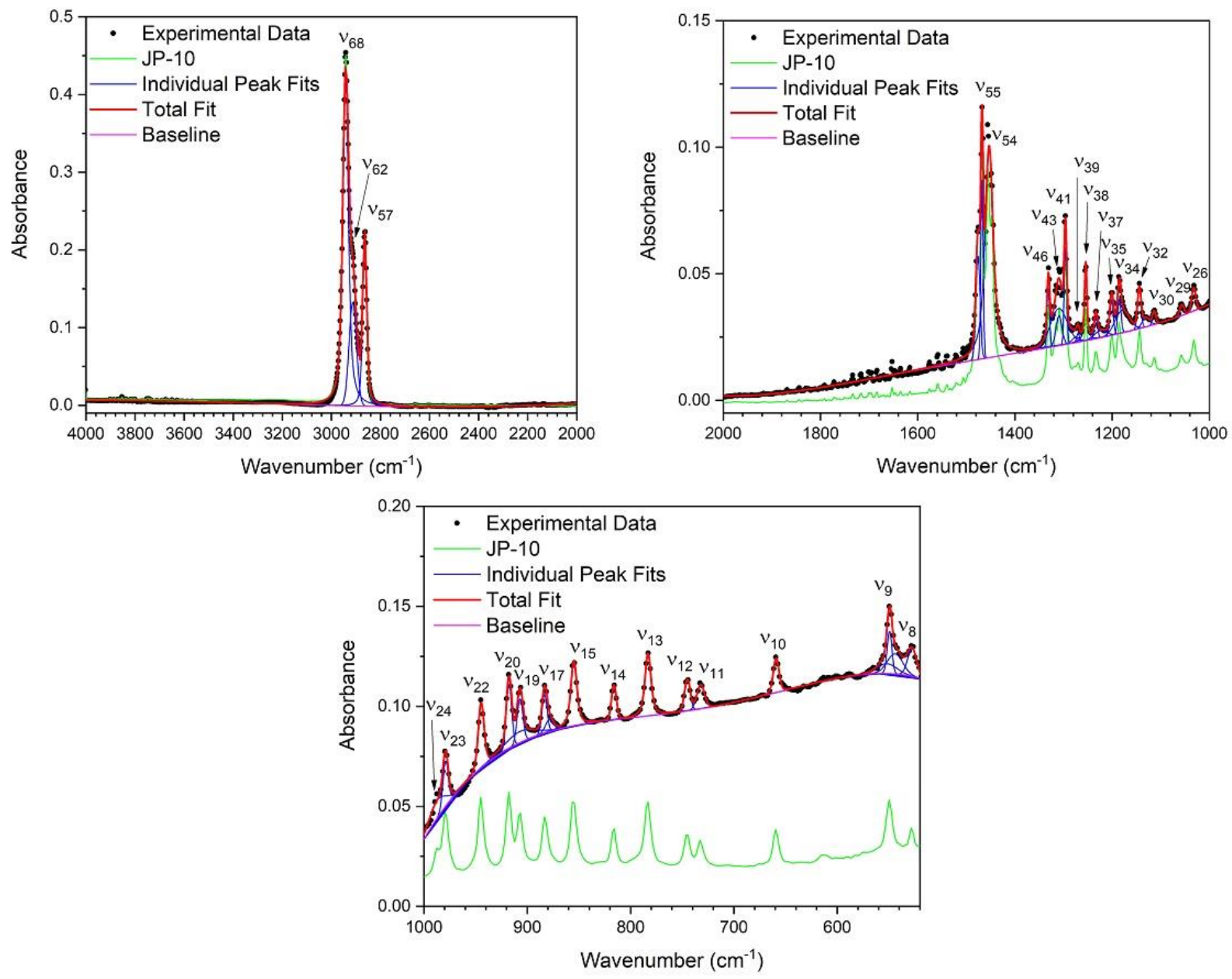

Figure S1. ATR-FTIR spectra of Al/JP-10 with the individual peak fits. FTIR spectra of pure JP10 (green lines) are shown for comparison. The individual peaks are labelled with $v_{\#}$ and the peak assignments are compiled in Table $\mathrm{S} 2$. 

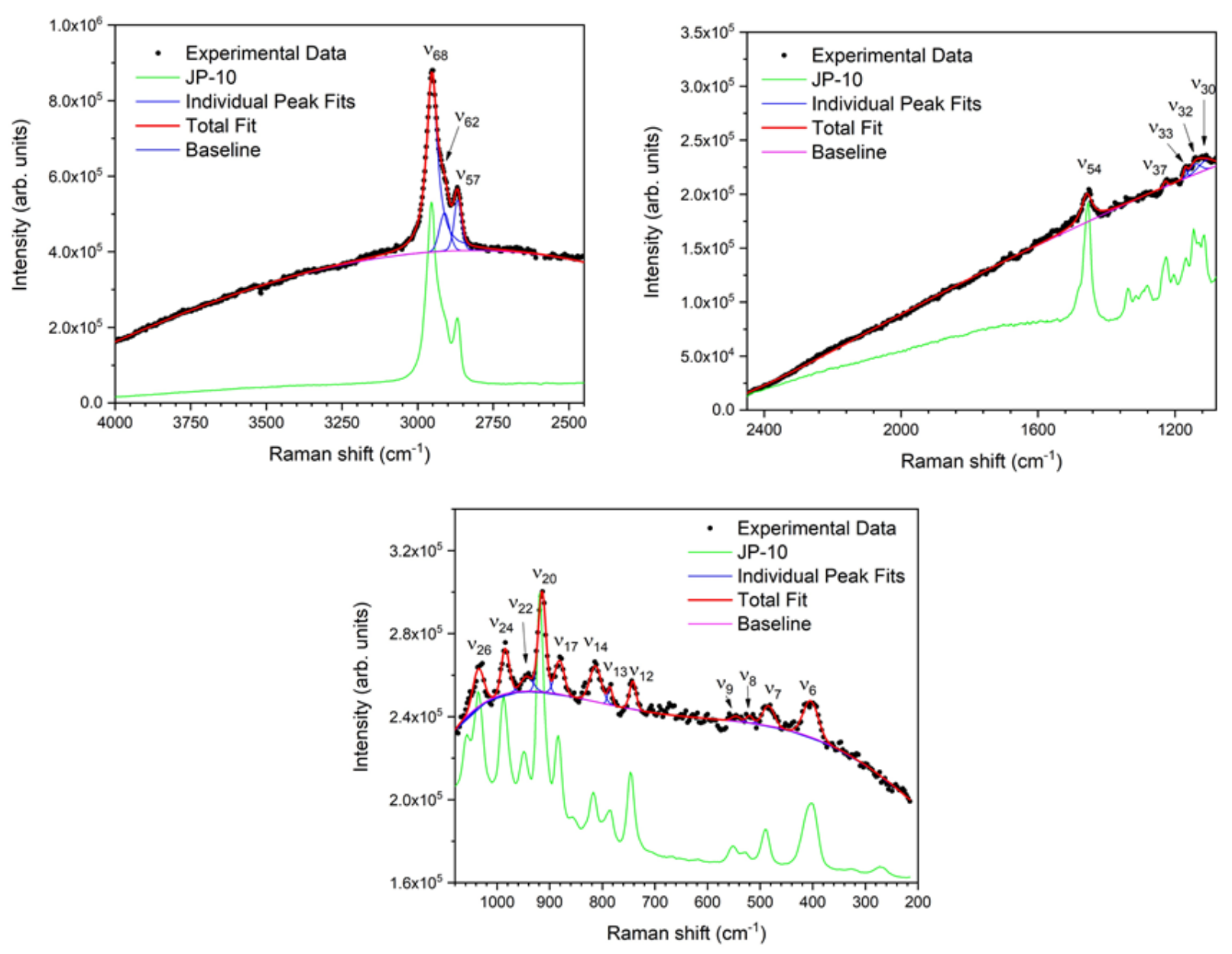

Figure S2. Raman spectra of A1/JP-10 with the individual peak fits. Raman spectra of pure JP-10 (green lines) are shown for comparison. The individual peaks are labelled with $\nu_{\#}$ and the peak assignments are compiled in Table $\mathbf{S} 2$. 

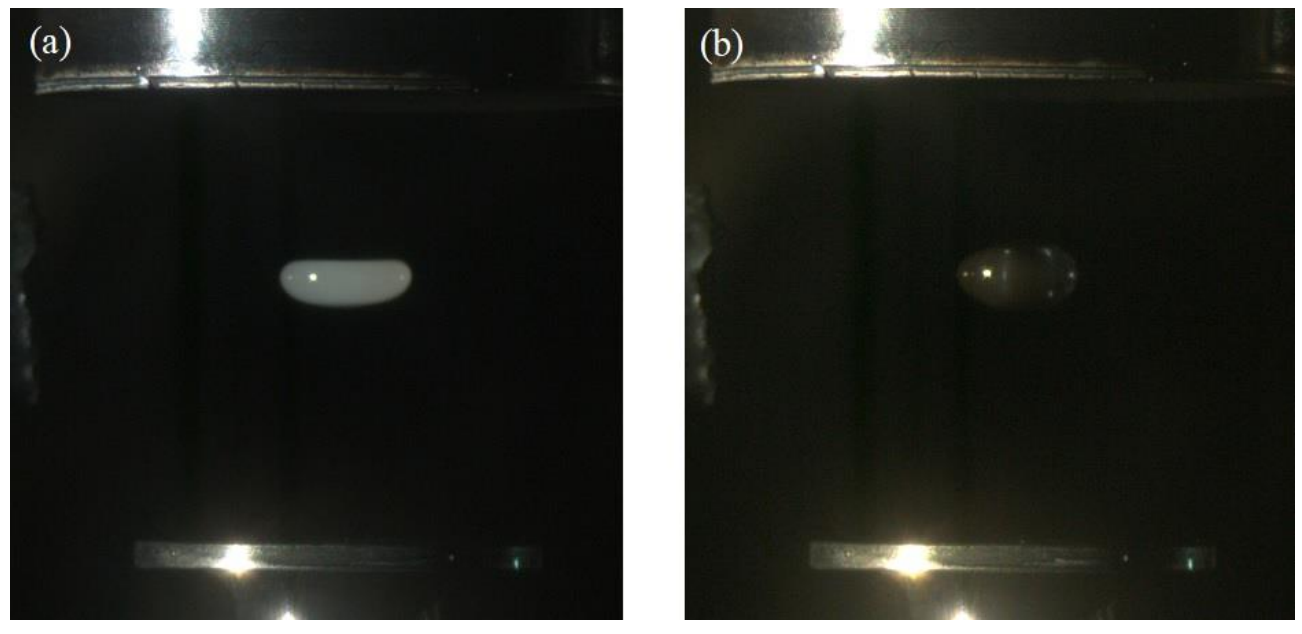

Figure S3. Photographs of a levitated droplet of (a) $\mathrm{Al}_{2} \mathrm{O}_{3} / \mathrm{JP}-10$ or (b) CuO/JP-10. 


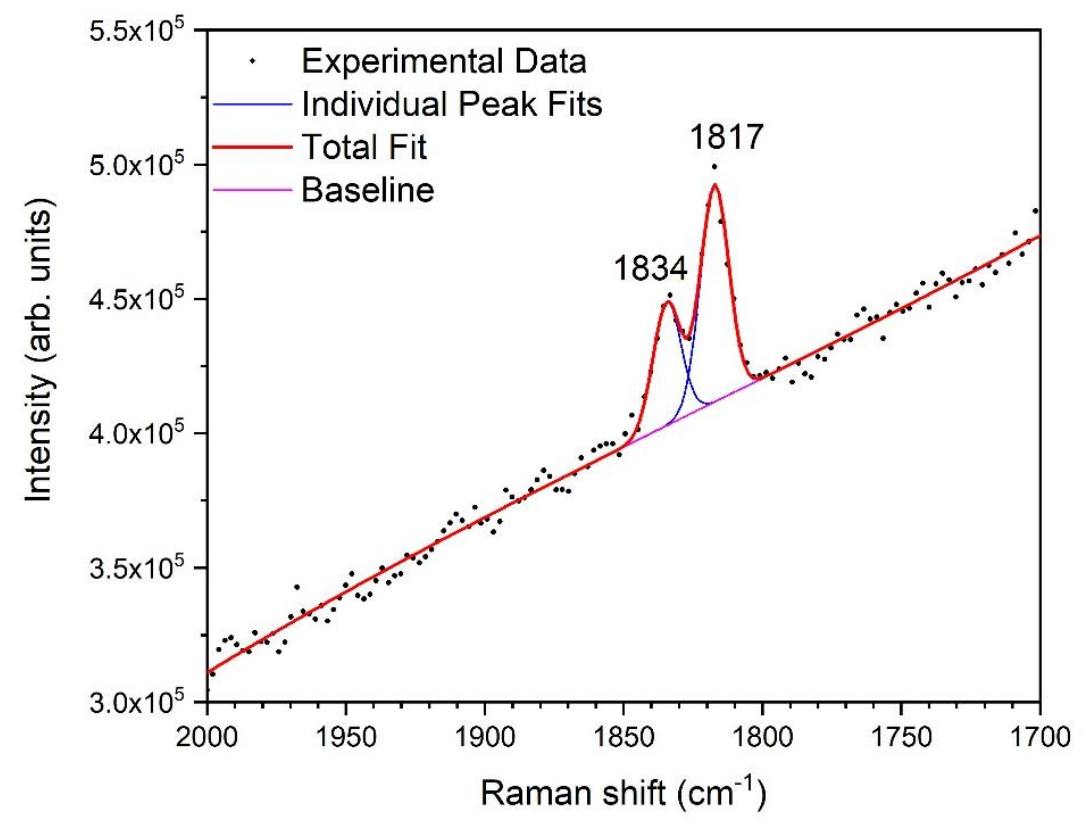

Figure S4. Emission doublet produced by the ${ }^{2} \mathrm{P}_{3 / 2,1 / 2} \rightarrow{ }^{2} \mathrm{~S}_{1 / 2}$ transitions of atomic sodium at $589.00 \mathrm{~nm}$ and $589.59 \mathrm{~nm}$, respectively, which appears in the Raman spectrum during ignition of an $\mathrm{Al} / \mathrm{JP}-10$ droplet levitated in $40 \% \mathrm{O}_{2}$ and $60 \% \mathrm{Ar}$. 
(a)

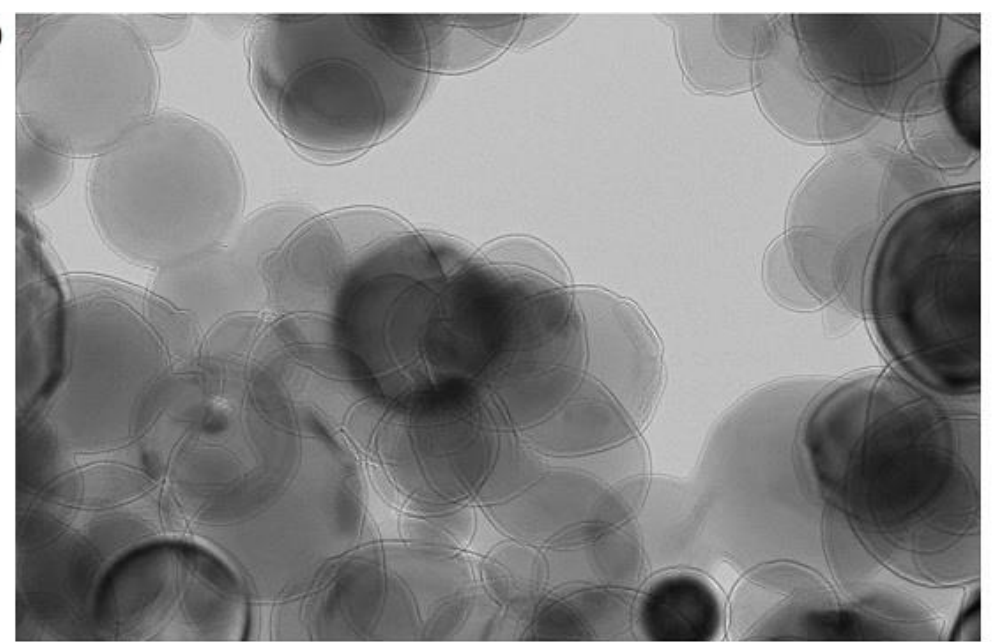

(b)

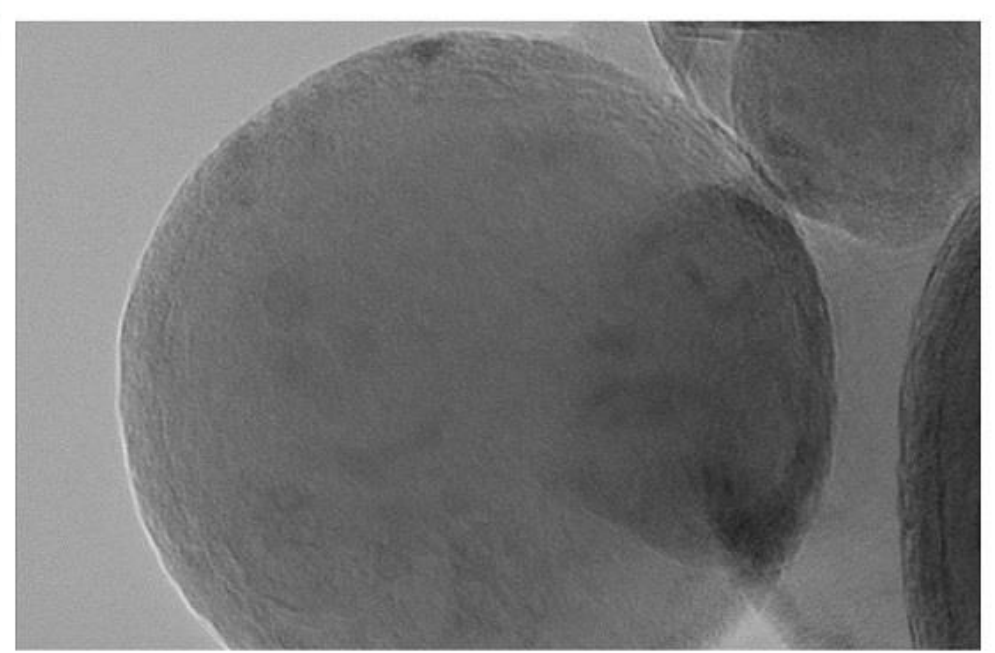

Figure S5. TEM images showing (a) the different sizes of the aluminum nanoparticles and (b) a single particle to display the $\mathrm{Al}_{2} \mathrm{O}_{3}$ outer shell. 


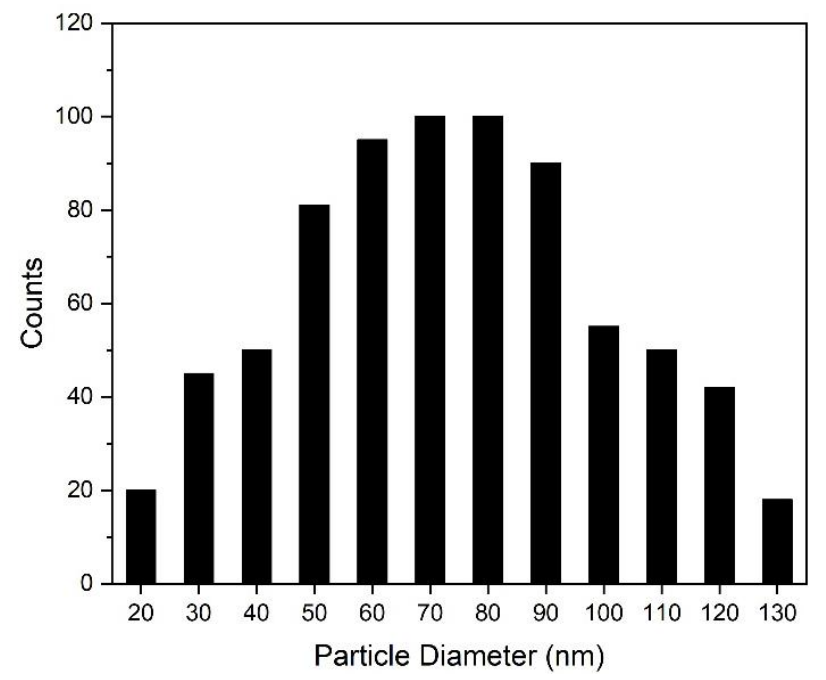

Figure S6. Size distribution of the aluminum nanoparticles. The counts are proportional to the volume of particles with diameters within the specified ranges. 

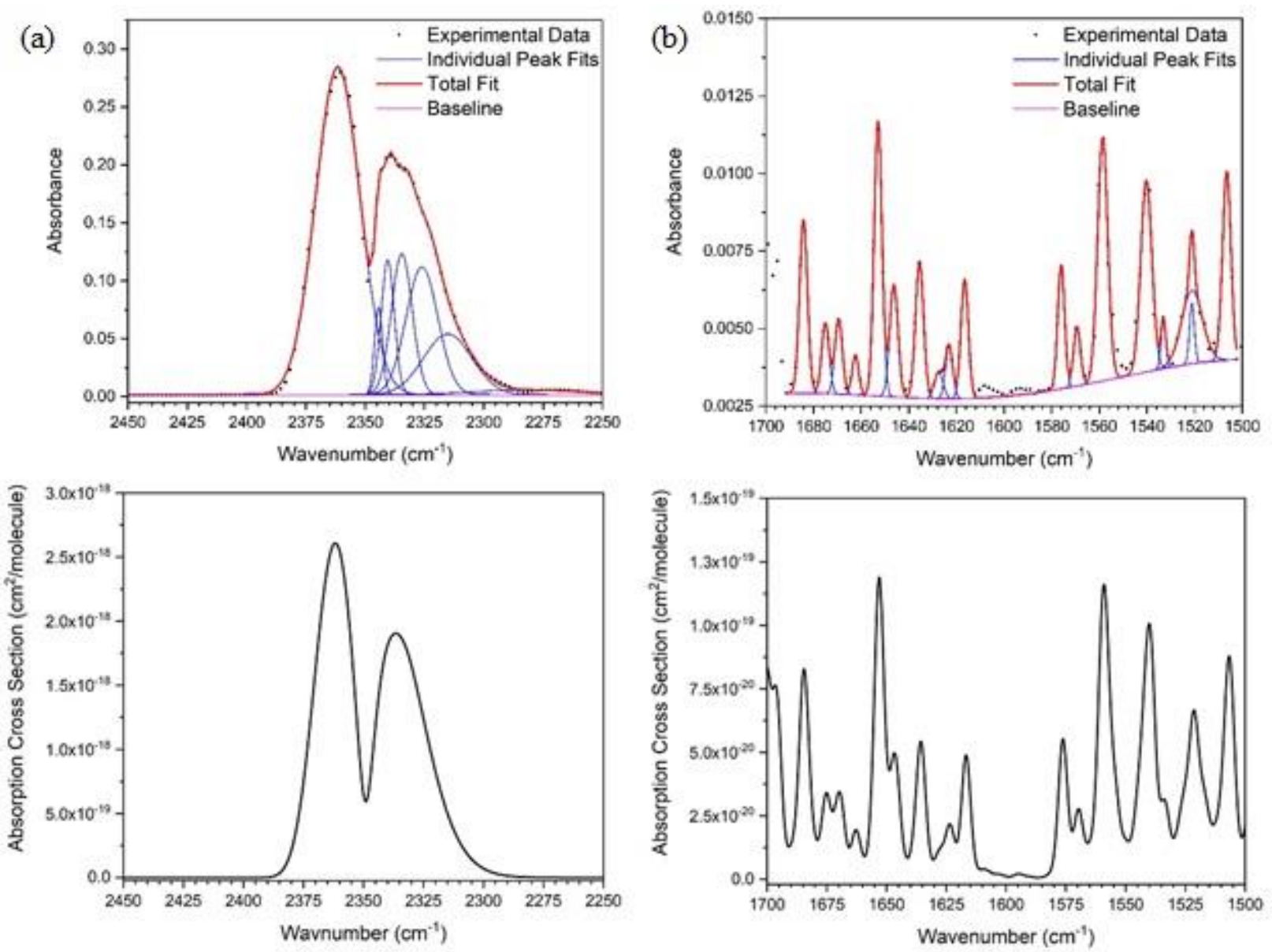

Figure S7. Experimental FTIR spectra (top row) and the reference absorption cross sections (bottom row). ${ }^{2}$ Column (a) shows the $2349 \mathrm{~cm}^{-1}$ absorption band c of $\mathrm{CO}_{2}$ (Table S3) and column (b) the $\mathrm{H}_{2} \mathrm{O}$ absorption band $\mathrm{d}$ (Table S4). The spectral resolution was $4 \mathrm{~cm}^{-1}$ in all the Figures. 
Table S1. Vibrational Mode Assignments for the Observed Peaks in the FTIR and Raman Spectra of JP-10.

\begin{tabular}{|c|c|c|c|c|}
\hline Number & $\begin{array}{c}\text { FTIR } \\
\text { Wavenumbers } \\
\left(\mathbf{c m}^{-1}\right)^{\mathbf{a}}\end{array}$ & $\begin{array}{c}\text { Raman } \\
\text { Wavenumbers } \\
\left(\mathbf{c m}^{-1}\right)^{\mathbf{a}}\end{array}$ & $\begin{array}{c}\text { Calculated } \\
\text { Wavenumbers } \\
\left(\mathbf{c m}^{-1}\right)^{\mathbf{b}}\end{array}$ & $\begin{array}{c}\text { Vibrational } \\
\text { Mode } \\
\text { Assignment }\end{array}$ \\
\hline 68 & 2942 & 2955 & 3073 & $\mathrm{CH}$ stretch \\
\hline 62 & 2913 & 2912 & 3042 & $\mathrm{CH}_{2}$ stretch \\
\hline 57 & 2865 & 2868 & 3016 & $\mathrm{CH}$ stretch \\
\hline 55 & 1468 & $1472 \pm 6$ & 1514 & $\mathrm{CH}_{2}$ scissor \\
\hline 54 & 1456 & 1454 & 1502 & $\mathrm{CH}_{2}$ scissor \\
\hline 47 & 1337 & 1343 & $\mathrm{CH}_{\text {wag }}$ \\
\hline 46 & 1330 & $1315 \pm 3$ & 1331 & $\mathrm{CH}_{2}$ wag \\
\hline 44 & 1316 & & 1311 & $\mathrm{CH}_{2}$ wag \\
\hline 43 & 1309 & $1300 \pm 8$ & 1301 & $\mathrm{CH}_{2}$ wag \\
\hline 41 & 1297 & 1284 & 1289 & $\mathrm{CH}_{\text {wag }}$ \\
\hline 40 & 1277 & & 1261 & $\mathrm{CH}_{2}$ wag \\
\hline 39 & 1270 & 1130 & 1251 & $\mathrm{CH}_{2}$ twist \\
\hline 38 & 1254 & 1228 & 1227 & $\mathrm{CH}_{2}$ twist \\
\hline 37 & 1233 & 1202 & 1205 & $\mathrm{CH}_{2}$ rock \\
\hline 35 & 1201 & & 1193 & $\mathrm{CH}_{2}$ twist \\
\hline 34 & 1186 & $\mathrm{CH}_{2}$ wag \\
\hline 33 & & 1145 & $\mathrm{CH}_{2}$ twist \\
\hline 32 & 1144 & & $\mathrm{CH}_{2}$ twist \\
\hline 31 & $1129 \pm 2$ & $\mathrm{CH}_{2}$ twist \\
\hline
\end{tabular}




\begin{tabular}{|c|c|c|c|c|}
\hline Number & $\begin{array}{c}\text { FTIR } \\
\text { Wavenumbers } \\
\left(\mathrm{cm}^{-1}\right)^{\mathrm{a}}\end{array}$ & $\begin{array}{c}\text { Raman } \\
\text { Wavenumbers } \\
\left(\mathrm{cm}^{-1}\right)^{\mathbf{a}}\end{array}$ & $\begin{array}{c}\text { Calculated } \\
\text { Wavenumbers } \\
\left(\mathrm{cm}^{-1}\right)^{\mathbf{b}}\end{array}$ & $\begin{array}{c}\text { Vibrational } \\
\text { Mode } \\
\text { Assignment }\end{array}$ \\
\hline 30 & 1113 & 1115 & 1073 & Rock, $\mathrm{CH}_{2}$ twist \\
\hline 29 & 1057 & 1057 & 1054 & $\mathrm{CH}_{2} \mathrm{Wag}$ \\
\hline 26 & 1032 & 1035 & 1041 & $\mathrm{CH}_{2}$ Twist \\
\hline 24 & 988 & 988 & 992 & CC stretch \\
\hline 23 & 980 & & 963 & CC stretch \\
\hline 22 & 945 & 948 & 955 & Ring breathing \\
\hline 20 & 918 & 919 & 918 & $\mathrm{CH}_{2}$ twist \\
\hline 19 & 907 & & 913 & CC stretch \\
\hline 17 & 883 & 884 & 889 & CC stretch \\
\hline 15 & 855 & 857 & 863 & CCC bend \\
\hline 14 & 816 & 817 & 828 & $\mathrm{CH}_{2}$ rock \\
\hline 13 & 783 & 785 & 792 & CC stretch \\
\hline 12 & 745 & 746 & 753 & CC stretch \\
\hline 11 & 733 & & 739 & CCC bend \\
\hline \multirow[t]{2}{*}{10} & 659 & & 671 & Ring rock \\
\hline & 613 & & & \\
\hline 9 & 554 & 552 & 554 & CCC bend \\
\hline 8 & 528 & $527 \pm 3$ & 534 & Ring twist \\
\hline 7 & & 490 & 495 & CCC bend \\
\hline 6 & & 396 & 397 & $\mathrm{CCC}$ bend \\
\hline
\end{tabular}

${ }^{a}$ The uncertainties are equal to, or less than, $1 \mathrm{~cm}^{-1}$ unless stated otherwise. ${ }^{\mathrm{b}} \mathrm{B}$ ased on reference 4 . 
Table S2. Vibrational Mode Assignments for the Observed Peaks in the FTIR and Raman Spectra of $\mathrm{Al} / \mathrm{JP}-10$.

\begin{tabular}{|c|c|c|c|c|}
\hline Number & $\begin{array}{c}\text { FTIR } \\
\text { Wavenumbers } \\
\left(\mathrm{cm}^{-1}\right)^{\mathrm{a}}\end{array}$ & $\begin{array}{c}\text { Raman } \\
\text { Wavenumbers } \\
\left(\mathrm{cm}^{-1}\right)^{\mathrm{a}}\end{array}$ & $\begin{array}{c}\text { Calculated } \\
\text { Wavenumbers } \\
\left(\mathrm{cm}^{-1}\right)^{\mathbf{b}}\end{array}$ & $\begin{array}{c}\text { Vibrational } \\
\text { Mode } \\
\text { Assignment }\end{array}$ \\
\hline 68 & 2942 & 2952 & 3073 & CH stretch \\
\hline 62 & 2913 & 2911 & 3042 & $\mathrm{CH}_{2}$ stretch \\
\hline 57 & 2864 & 2868 & 3016 & $\mathrm{CH}$ stretch \\
\hline 55 & 1468 & & 1514 & $\mathrm{CH}_{2}$ scissor \\
\hline 54 & 1453 & 1457 & 1502 & $\mathrm{CH}_{2}$ scissor \\
\hline 47 & & 1337 & 1343 & CH wag \\
\hline 46 & 1331 & & 1331 & $\mathrm{CH}_{2}$ wag \\
\hline 43 & $1309 \pm 4$ & & 1311 & $\mathrm{CH}_{2}$ wag \\
\hline 41 & 1296 & & 1301 & CH wag \\
\hline 39 & $1270 \pm 18$ & & 1261 & $\mathrm{CH}_{2}$ twist \\
\hline 38 & 1254 & & 1251 & $\mathrm{CH}_{2}$ twist \\
\hline 37 & $1233 \pm 2$ & $1228 \pm 2$ & 1227 & $\mathrm{CH}_{2}$ rock \\
\hline 35 & 1201 & & 1205 & $\mathrm{CH}_{2}$ twist \\
\hline 34 & $1185 \pm 2$ & & 1193 & $\mathrm{CH}_{2}$ wag \\
\hline 33 & & $1170 \pm 2$ & 1169 & $\mathrm{CH}_{2}$ twist \\
\hline 32 & 1144 & $1141 \pm 23$ & 1153 & $\mathrm{CH}_{2}$ twist \\
\hline 30 & $1113 \pm 4$ & $1108 \pm 71$ & 1073 & $\mathrm{CH}_{2}$ Rock, twist \\
\hline 29 & $1056 \pm 2$ & & 1054 & $\mathrm{CH}_{2} \mathrm{Wag}$ \\
\hline 26 & 1032 & 1036 & 1041 & $\mathrm{CH}_{2}$ Twist \\
\hline 24 & 987 & 984 & 992 & CC stretch \\
\hline 23 & 979 & & 963 & CC stretch \\
\hline 22 & 945 & $944 \pm 2$ & 955 & Ring breathing \\
\hline
\end{tabular}




\begin{tabular}{|c|c|c|c|c|}
\hline Number & $\begin{array}{c}\text { FTIR } \\
\text { Wavenumbers } \\
\left(\mathbf{c m}^{-1}\right)^{\mathbf{a}}\end{array}$ & $\begin{array}{c}\text { Raman } \\
\text { Wavenumbers } \\
\left(\mathrm{cm}^{-1}\right)^{\mathrm{a}}\end{array}$ & $\begin{array}{c}\text { Calculated } \\
\text { Wavenumbers } \\
\left(\mathbf{c m}^{-1}\right)^{\mathbf{b}}\end{array}$ & $\begin{array}{l}\text { Vibrational } \\
\text { Mode } \\
\text { Assignment }\end{array}$ \\
\hline 20 & 918 & 915 & 918 & $\mathrm{CH}_{2}$ twist \\
\hline 19 & 907 & & 913 & CCC stretch \\
\hline 17 & $883 \pm 2$ & 881 & 889 & CC stretch \\
\hline 15 & 855 & & 863 & CCC bend \\
\hline 14 & 816 & 812 & 828 & $\mathrm{CH}_{2}$ rock \\
\hline 13 & 783 & 785 & 792 & CC stretch \\
\hline 12 & 745 & 741 & 753 & CC stretch \\
\hline 11 & 732 & & 739 & CCC bend \\
\hline 10 & 659 & & 671 & Ring rock \\
\hline 9 & 549 & $545 \pm 6$ & 554 & CCC bend \\
\hline 8 & 528 & $520 \pm 4$ & 534 & Ring twist \\
\hline 7 & & 484 & 495 & CCC bend \\
\hline 6 & & 404 & 397 & CCC bend \\
\hline
\end{tabular}

${ }^{a}$ The uncertainties are equal to, or less than, $1 \mathrm{~cm}^{-1}$ unless stated otherwise.

${ }^{b}$ Based on reference ${ }^{4}$. 
Table S3. Assignment of the vibrational modes in the FTIR spectra collected following oxidation of Al/JP-10.

\begin{tabular}{|c|c|c|c|c|c|c|}
\hline Band & $\begin{array}{c}\text { Experimental } \\
\text { band center } \\
\left(\mathbf{c m}^{-1}\right)\end{array}$ & $\begin{array}{c}\text { Reference band } \\
\left.\text { center } \mathbf{( c m}^{-\mathbf{1}}\right)\end{array}$ & Molecule & $\begin{array}{c}\text { Number } \\
\text { (symmetry) }\end{array}$ & $\begin{array}{c}\text { Vibrational } \\
\text { Mode }\end{array}$ & Ref. \\
\hline $\mathrm{a}$ & 3714 & 3715 & $\mathrm{CO}_{2}$ & $\begin{array}{c}v_{1}\left(\sigma_{\mathrm{g}}{ }^{+}\right)+ \\
v_{3}\left(\sigma_{\mathrm{u}}{ }^{+}\right)\end{array}$ & $\begin{array}{c}\text { Combination } \\
\text { band }\end{array}$ & 5 \\
\hline $\mathrm{b}$ & 3612 & 3613 & $\mathrm{CO}_{2}$ & $v_{1}\left(\sigma_{\mathrm{g}}{ }^{+}\right)+$ & $\begin{array}{c}\text { Combination } \\
v_{3}\left(\sigma_{\mathrm{u}}{ }^{+}\right)\end{array}$ & 5 \\
\hline $\mathrm{c}$ & 2349 & 2349 & $\mathrm{CO}_{2}$ & $v_{3}\left(\sigma_{\mathrm{u}}{ }^{+}\right)$ & $\mathrm{CO}_{2}$ asym. str. & 5 \\
\hline $\mathrm{d}$ & 1635 & 1635 & $\mathrm{H}_{2} \mathrm{O}$ & $v_{2}\left(\mathrm{~A}^{\prime}\right)$ & $\mathrm{OH}$ bend & 6 \\
\hline $\mathrm{e}$ & 669 & 667 & $\mathrm{CO}_{2}$ & $v_{2}\left(\pi_{\mathrm{u}}\right)$ & $\mathrm{CO}_{2}$ bend & 5 \\
\hline
\end{tabular}

Movie M1. High-speed video of an igniting Al/JP-10 droplet. 


\section{REFERENCES}

(1) Bruno, T. J.; Huber, M. L.; Laesecke, A.; Lemmon, E. W.; Perkins, R. A., Thermochemical and Thermophysical Properties of JP-10. 2006, 6640, 325.

(2) Mikhailenko, C. N.; Babikov, Y. L.; Golovko, V. F., Information-Calculating System Spectroscopy of Atmospheric Gases. The Structure and Main Functions. J Atmos. Oceanic Opt. 2005, 18, 685-695.

(3) Gordon, I. E.; Rothman, L. S.; Hill, C.; Kochanov, R. V.; Tan, Y.; Bernath, P. F.; Birk, M.; Boudon, V.; Campargue, A.; Chance, K. V.; Drouin, B. J.; Flaud, J. M.; Gamache, R. R.; Hodges, J. T.; Jacquemart, D.; Perevalov, V. I.; Perrin, A.; Shine, K. P.; Smith, M. A. H.; Tennyson, J.; Toon, G. C.; Tran, H.; Tyuterev, V. G.; Barbe, A.; Csaszar, A. G.; Devi, V. M.; Furtenbacher, T.; Harrison, J. J.; Hartmann, J. M.; Jolly, A.; Johnson, T. J.; Karman, T.; Kleiner, I.; Kyuberis, A. A.; Loos, J.; Lyulin, O. M.; Massie, S. T.; Mikhailenko, S. N.; Moazzen-Ahmadi, N.; Muller, H. S. P.; Naumenko, O. V.; Nikitin, A. V.; Polyansky, O. L.; Rey, M.; Rotger, M.; Sharpe, S. W.; Sung, K.; Starikova, E.; Tashkun, S. A.; Vander Auwera, J.; Wagner, G.; Wilzewski, J.; Wcislo, P.; Yu, S.; Zak, E. J., The HITRAN2016 Molecular Spectroscopic Database. J. Quant. Spectrosc. Radiat. Transfer 2017, 203, 3-69.

(4) Morozov, A. N.; Mebel, A. M.; Kaiser, R. I., A Theoretical Study of Pyrolysis of exoTetrahydrodicyclopentadiene and Its Primary and Secondary Unimolecular Decomposition Products. J. Phys. Chem. A 2018, 122, 4920-4934.

(5) Miller, C. E.; Brown, L. R., Near Infrared Spectroscopy of Carbon Dioxide $\mathrm{I} .{ }^{16} \mathrm{O}^{12} \mathrm{C}^{16} \mathrm{O}$ Line Positions. J. Mol. Spectrosc. 2004, 228, 329-354.

(6) Zou, Q.; Varanasi, P., Laboratory Measurement of the Spectroscopic Line Parameters of Water Vapor in the 610-2100 and 3000-4050 $\mathrm{cm}^{-1}$ Regions at Lower-Tropospheric Temperatures. J. Quant. Spectrosc. Radiat. Transfer 2003, 82, 45-98. 